\title{
Photonic Band Structure: The Face-Centered-Cubic Case Employing Nonspherical Atoms
}

\author{
E. Yablonovitch and T. J. Gmitter \\ Bell Communications Research, Navesink Research Center, Red Bank, New Jersey 07701-7040
}

\author{
K. M. Leung \\ Department of Physics, Polytechnic University, Brooklyn, New York 11201
}

(Received 26 December 1990)

\begin{abstract}
We introduce a practical, new, face-centered-cubic dielectric structure which simultaneously solves two of the outstanding problems in photonic band structure. In this new "photonic crystal" the atoms are nonspherical, lifting the degeneracy at the $W$ point of the Brillouin zone, and permitting a full photonic band gap rather than a pseudogap. Furthermore, this fully three-dimensional fcc structure lends itself readily to microfabrication on the scale of optical wavelengths. It is created by simply drilling three sets of holes $35.26^{\circ}$ off vertical into the top surface of a solid slab or wafer, as can be done, for example, by chemical-beam-assisted ion etching.
\end{abstract}

PACS numbers: 42.50.-p, 41.10.Hv, 71.25.Cx, 84.90.+a

There is an interesting analogy between electron waves in a crystal and light waves in a three-dimensionally periodic dielectric structure. Both should be described $[1,2]$ by band theory. The concepts of reciprocal space, Brillouin zones, dispersion relations, Bloch wave functions, Van Hove singularities, etc., are now being applied [3-6] to optical waves.

A search has been underway for three-dimensional topologies in which a "photonic band gap" can open up. This would be an energy band in which optical modes, spontaneous emission, and zero-point fluctuations are all absent. Indeed, a photonic band gap would be essentially ideal since optical dielectric response can be real and dissipationless.

In addition to the obvious applications in atomic physics, inhibited spontaneous emission can now begin to play a role in semiconductor and solid-state electronics. If the photonic band gap overlaps the electronic band edge, spontaneous electron-hole recombination is rigorously forbidden. In a semiconductor laser, this would lead to near unity quantum efficiency into the lasing mode. Photon-number-state squeezing [7] into that mode would be greatly enhanced.

There have been two main challenges in this field. The first was to show that a full three-dimensional photonic band gap could actually exist in some type of dielectric structure. The second was to show that such a forbidden gap could be created in a microstructure amenable to practical microfabrication.

At the outset it was realized [1] that a face-centeredcubic (fcc) array in real space would produce the "roundest" Brillouin zone in reciprocal space. Such a spherelike Brillouin zone improves the prospects for a forbidden gap to overlap all the way around its surface. But it was unclear what should be the real-space shape of the atoms in the fcc array. The original suggestion [1] called for cubic atoms. Later, the first experimental effort [8] concentrated on dielectric spheres and on spherical voids in a dielectric background. The spherical-void structure appeared to perform particularly well.

During this same period, electronic band theorists be- gan calculating photonic band structure. It rapidly became apparent that the familiar scalar-wave-band theory, so frequently used for electrons in solids, was in utter disagreement with experiment on photons [9-12]. Recently, a full vector-wave-band theory [3-5] became available, which not only agreed with experiment, but highlighted some discrepancies in experiment. Vectorwave-band theory showed that spherical atomic symmetry produced a degeneracy between valence and conduction bands at the $W$ point of the Brillouin zone, allowing only a pseudogap, rather than a full photonic band gap. Ho, Chan, and Soukoulis [5] were the first to overcome this problem. They introduced the diamond structure, which required two atoms per fcc unit cell.

More generally we find that the symmetry-induced degeneracy in fcc structures is lifted by making the atoms nonspherical. This has led to a practical, new fcc structure which lends itself readily to microfabrication on the scale of optical wavelengths. It is created by simply drilling three sets of holes $35.26^{\circ}$ off vertical into the top surface of a solid slab or wafer, as can be done, for example, by reactive ion etching. At refractive index $n \sim 3.6$, typical of semiconductors, the 3D forbidden photonic bandgap width in this new structure is $\sim 20 \%$ of its center frequency. Calculations indicate that the gap remains open for refractive indices $n \gtrsim 2.1$.

The Wigner-Seitz (WS) unit cell of the fcc lattice is a rhombic dodecahedron as shown in Fig. 1. The problem of creating an arbitrary fcc dielectric structure reduces to the problem of filling the fcc WS real-space unit cell with an arbitrary spatial distribution of dielectric material. Real space is then filled by repeated translation and close packing of the WS unit cells.

Figure 1(a) shows a WS unit cell filled by an oversized spherical void, a structure which performed rather well in Ref. [8]. Since the spheres were slightly larger than close packed, the voids broke through the surfaces of the WS unit cell as indicated by the dashed circles on the faces of the rhombic dodecahedron in Fig. 1(a). In Ref. [8] it was already pointed out that there was a symmetryinduced degeneracy at the $W$ point of the Brillouin zone 


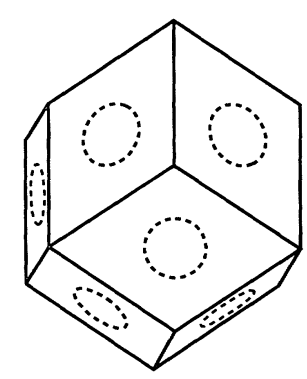

(a)

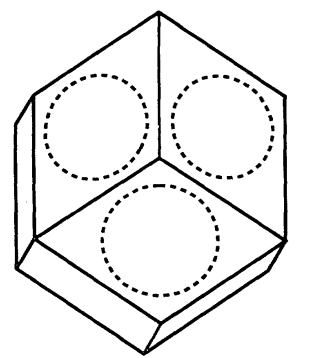

(b)

FIG. 1. The Wigner-Seitz real-space unit cell of the fcc lattice is a rhombic dodecahedron. In Ref. [8], slightly oversized spherical voids were inscribed into the unit cell, breaking through the faces, as illustrated by the dashed lines in (a). The current structure, shown in (b), is nonspherical. Cylindrical holes are drilled through the top three facets of the rhombic dodecahedron and exit through the bottom three facets. The resulting atoms are roughly cylindrical, and have a preferred axis in the vertical direction.

in fcc structures. There was a danger that the valence and conduction bands could touch at the degeneracy, closing the photonic band gap. Based on the weight of experimental evidence, however, Ref. [8] argued that the degeneracy had only caused adjacent conduction-band levels to touch, permitting the gap to remain open. Vector-wave-band theory, which has become quite successful recently [3-5], contradicted this. It showed that the degeneracy did indeed cause valence and conduction bands to touch at $W$, permitting only a pseudogap rather than a full photonic band gap. Unfortunately, the finitesized experimental sample in Ref. [8] allowed inadequate resolution to detect touching at isolated points on the Brillouin zone.

We have made a close examination [13] of the degenerate wave functions at $W$ in the nearly free-photon model, and learned that a distortion of the spherical atoms along the $\langle 111\rangle$ direction will lift the degeneracy. The diamond structure of Ho, Chan, and Soukoulis [5], with two atoms per unit cell, fulfills this function admirably. Alternatively, the WS unit cell in Fig. 1(b) also has great merit for this purpose. Holes are drilled through the top three facets of the rhombic dodecahedron and exit through the bottom three facets. The beauty of the structure in Fig. 1(b) is that a stacking of WS unit cells results in straight holes which pass clear through the entire

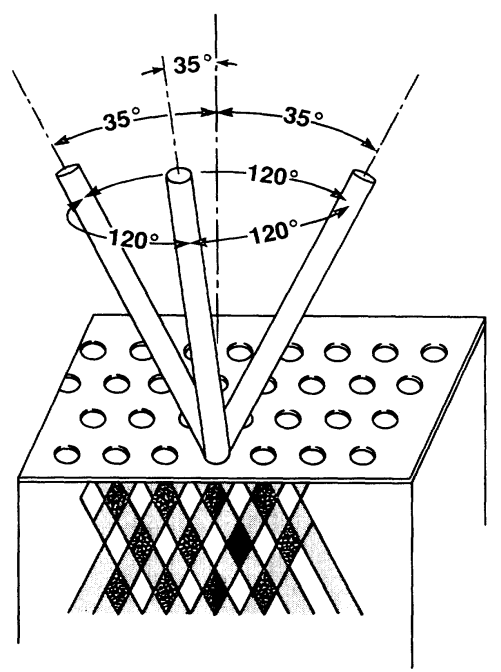

FIG. 2. The method of constructing an fcc lattice of the Wigner-Seitz cells as shown in Fig. 1(b). A slab of material is covered by a mask consisting of a triangular array of holes. Each hole is drilled through 3 times, at an angle $35.26^{\circ}$ away from normal, and spread out $120^{\circ}$ on the azimuth. The resulting crisscross of holes below the surface of the slab, suggested by the cross hatching shown here, produces a fully threedimensionally periodic fcc structure, with unit cells as given by Fig. 1(b). The drilling can be done by a real drill bit for microwave work, or by reactive ion etching to create an fcc structure at optical wavelengths.

"crystal." The "atoms" are odd-shaped, roughly cylindrical voids centered in the WS unit cell, with a preferred axis pointing to the top vertex, $\langle 111\rangle$.

An operational illustration of the construction which produces an fcc crystal of such WS unit cells is shown in Fig. 2. A slab of material is covered by a mask containing a triangular array of holes. Three drilling operations are conducted through each hole, $35.26^{\circ}$ off normal incidence and spread out $120^{\circ}$ on the azimuth. The resulting crisscross of holes below the surface of the slab produces a fully three-dimensionally periodic fcc structure, with WS unit cells given by Fig. 1(b). The drilling can be done by a real drill bit for microwave work, or by reactive ion etching to create an fcc structure at optical wavelengths. We have fabricated such crystals in the microwave region by direct drilling into a commercial, lowloss, dielectric material, Emerson \& Cumming Stycast12. Its microwave refractive index, $n \sim 3.6$, is meant to correspond to that of the common semiconductors, $\mathrm{Si}$, $\mathrm{GaAs}$, etc. By simply scaling down the dimensions, this structure can be employed equally well at optical wavelengths. In this paper we will present the measured and calculated, $\omega$ vs $k$, dispersion relations for this new photonic crystal.

The diamond symmetry of Ho, Chan, and Soukoulis [5] can be created by supplementing the operations of Fig. 2 with three additional drilling operations, making a 
total of six drilling directions. These three new drilling directions, $120^{\circ}$ apart, would all lie within the plane of the slab. Therefore they are somewhat difficult to implement experimentally. The six drilling directions correspond to the six inequivalent $\langle 110\rangle$ channeling holes in diamond structure.

We have experimentally surveyed three fcc structures, drilled in accordance with Fig. 2, to different ratios $d / a$ of hole diameter $d$ to fcc unit cube length $a$ : $d / a=0.361$, 0.433 , and 0.469 . The removed volume fraction was approximately $62 \%, 70 \%$, and $78 \%$ in the three cases, respectively. The $78 \%$-empty structure had the largest forbidden gap in this set and in this Letter we will present results on that structure only. We believe $78 \%$ is near the optimal volume fraction for this fcc geometry.

Our procedure is similar to the one we used in Ref. [8], except that our dynamic range was improved by using an HP-8510 Network Analyzer for all the measurements. The experimental aim is to fully explore all $4 \pi \mathrm{sr}$ in reciprocal space, while scanning frequency. The valenceband edge frequency is defined by a sudden drop in microwave transmission, while the conduction-band edge is defined by the frequency at which the transmitted signal recovers. Conservation of wave-vector momentum parallel to the slab entry face determines the band-edge position along the surface of the Brillouin zone. Since there are two polarizations, we can usually determine the two valence-band edges and two of the conduction bands.

Sometimes the coupling of external plane waves to internal Bloch waves is poor, and the experiment can miss one of the conduction-band edges, as happened in Ref. [8]. Finite sample size limits the usable dynamic range, exacerbating the weak-coupling problem. Therefore it is important to back up the measurements with numerical calculations, as we have done here. The scalar dielectric constant distribution of Fig. 1 is expanded as a Fourier series in reciprocal space, while Maxwell's equations are expanded [3] as vector plane waves. The eigenvalues converge after a few hundred plane waves are summed in the expansion.

In spite of the nonspherical atoms in Fig. 1(b), the Brillouin zone (BZ) is identical to the standard fcc BZ shown in textbooks. Nevertheless, we have chosen an unusual perspective from which to view the Brillouin zone in Fig. 3(a). Instead of having the fcc $\mathrm{BZ}$ resting on one of its diamond-shaped facets as is usually done, we have chosen in Fig. 3(a) to present it resting on a hexagonal face. Since there is a preferred axis for the atoms, the distinctive $L$ points centered in the top and bottom hexagons are threefold symmetry axes, and are labeled $L_{3}$. The $L$ points centered in the other six hexagons are symmetric only under a $360^{\circ}$ rotation, and are labeled $L_{1}$. It is helpful to know that the $U_{3}-K_{3}$ points are equivalent since they are a reciprocal-lattice vector apart. Likewise the $U_{1}-K_{1}$ points are equivalent.

Normal incidence on the slab of Fig. 2 sends the propagation vector directly toward $L_{3}$ in reciprocal space ("the
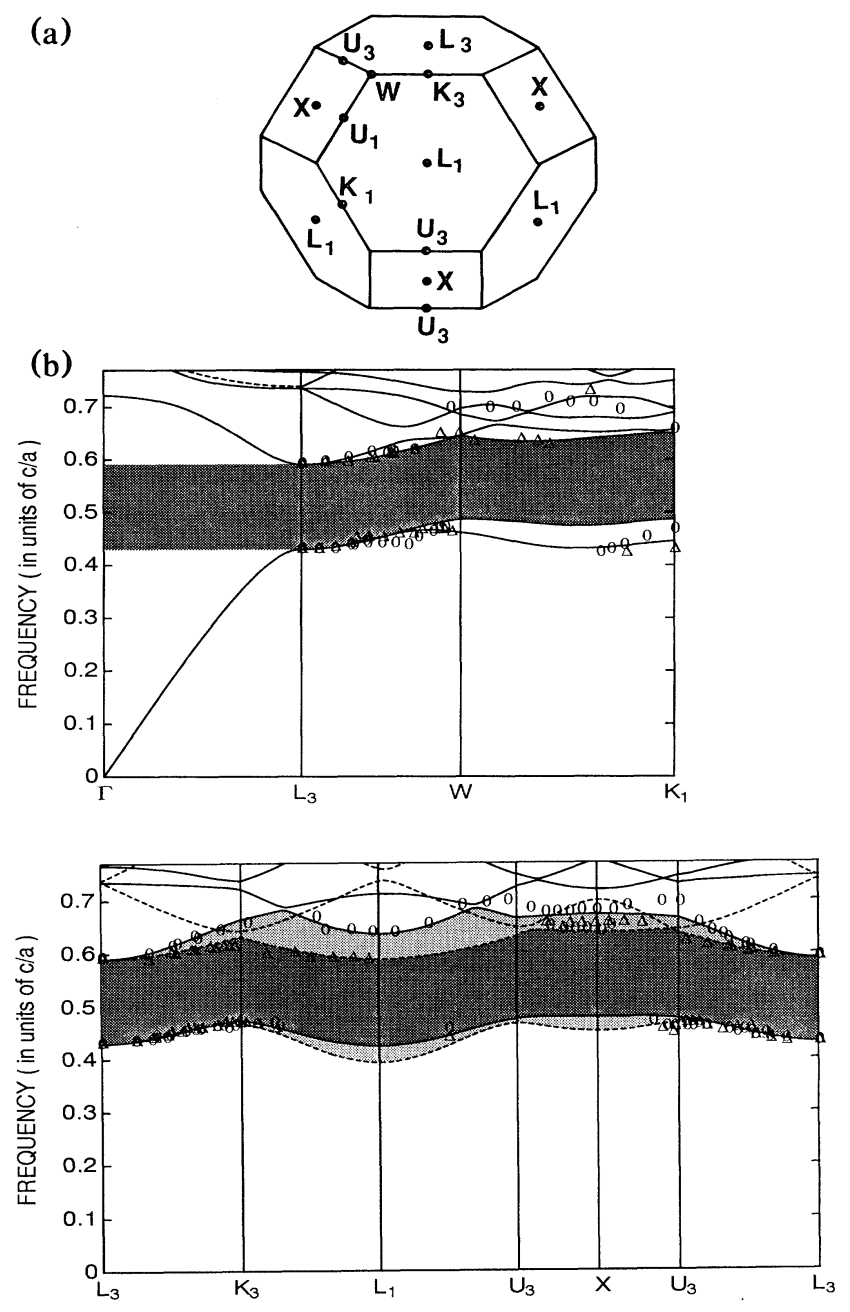

FIG. 3. (a) The Brillouin zone of an fcc structure incorporating nonspherical atoms, as in Fig. 1(b). Since the space lattice is not distorted, this is simply the standard fcc Brillouin zone lying on a hexagonal face rather than the usual cubic face. Only the $L$ points on the top and bottom hexagons are threefold symmetry axes. Therefore they are labeled $L_{3}$. The $L$ points on the other six hexagons are labeled $L_{1}$. The $U_{3}-K_{3}$ points are equivalent since they are a reciprocal-lattice vector apart. Likewise, the $U_{1}-K_{1}$ points are equivalent. (b) Frequency vs wave vector, $\omega$ vs $k$, dispersion along the surface of the Brillouin zone shown in (a), where $c / a$ is the speed of light divided by the fcc cube length. The ovals and triangles are the experimental points for $s$ and $p$ polarizations, respectively. The solid and dashed lines are the calculations for $s$ and $p$ polarizations, respectively. The dark shaded band is the totally forbidden band gap. The lighter shaded stripes above and below the dark band are forbidden only for $s$ and $p$ polarizations, respectively.

north pole"). Tilting the angle of incidence moves the propagation vector along a "meridian" toward the "equator." By choosing different azimuthal angles toward which to tilt, the full reciprocal space can be explored. Figure 3(b) shows the dispersion relations along different meridians for our primary experimental sample of nor- 
malized hole diameter $d / a=0.469$ and $78 \%$ volume fraction removed. The oval points represent experimental data with $s$ polarization (perpendicular to the plane of incidence, parallel to the slab surface), while the triangular points represent $p$ polarization (parallel to the plane of incidence, partially perpendicular to the slab surface). The horizontal abscissa in the lower graph of Fig. 3(b) $L_{3}-K_{3}-L_{1}-U_{3}-X-U_{3}-L_{3}$ represents a full meridian from the north pole to the south pole of the BZ. Along this meridian the Bloch wave functions separate neatly into $s$ and $p$ polarizations. The $s$ - and $p$-polarized theory curves are the solid and dashed lines, respectively. The dark shaded band is the totally forbidden photonic band gap. The lighter shaded stripes above and below the dark band are forbidden only for $s$ and $p$ polarizations, respectively.

Along the meridian $L_{3}-W-K_{1}$, the polarizations do not separate neatly, and only the totally forbidden photonic band gap is shaded. The top of the valence band is at $W$ and is mostly $s$ polarized, but the valence-band peaks at $U_{3}, X$, and $U_{1}$ are almost as high. The bottom of the conduction band is at $L_{1}$, purely $p$ polarized, is only marginally lower than the valley at $L_{3}$. We have also measured the imaginary wave-vector dispersion within the forbidden gap. At midgap we find an attenuation of $10 \mathrm{~dB}$ per unit cube length $a$. Therefore the photonic crystal need not be very many layers thick to effectively expel the zero-point electromagnetic field.

At a typical semiconductor refractive index, $n=3.6$, the 3D forbidden gap width is $19 \%$ of its center frequency. We have repeated the calculation at lower refractive indices, reoptimizing the hole diameter. Our calculations indicate that the gap remains open for refractive indices as low as $n=2.1$ using circular holes as in Fig. 2. In reactive ion etching, the projection of circular mask openings at $35^{\circ}$ leaves oval holes in the material, which might not perform as well. Fortunately we found, defying Murphy's law, that the forbidden gap width for oval holes is actually improved, fully $21.7 \%$ of its center frequency.

In the visible region, there are many transparent optical materials available with a refractive index above 2.1.
Furthermore, state-of-the-art reactive ion etching [14] can produce holes that are $\gtrsim 20$ times deeper than their diameter, deep enough to produce an fcc photonic crystal with substantial inhibition in the forbidden gap. It appears that the application of photonic band gaps to semiconductor physics, optical, and atomic physics may soon be practical.

E.Y. would like to thank the authors of Ref. [5] for telling about their diamond structure calculations before publication, and for intensive discussions of the degenerate Bloch wave functions at the $W$ point. John Gural deserves special thanks for his patience, dedication, and skilled machining of tens of thousands of holes, which made this project possible. K.M.L.'s work is supported by ONR Contract No. N00014-88-0500.

[1] E. Yablonovitch, Phys. Rev. Lett. 58, 2059 (1987).

[2] S. John, Phys. Rev. Lett. 58, 2486 (1987).

[3] K. M. Leung and Y. F. Liu, Phys. Rev. Lett. 65, 2646 (1990).

[4] Z. Zhang and S. Satpathy, Phys. Rev. Lett. 65, 2650 (1990).

[5] K. M. Ho, C. T. Chan, and C. M. Soukoulis, Phys. Rev. Lett. 65, 3152 (1990).

[6] S. John and J. Wang, Phys. Rev. Lett. 64, 2418 (1990).

[7] Y. Yamamoto and S. Machida, Phys. Rev. A 35, 5114 (1987).

[8] E. Yablonovitch and T. J. Gmitter, Phys. Rev. Lett. 63, 1950 (1989).

[9] S. Satpathy, Z. Zhang, and M. R. Salehpour, Phys. Rev. Lett. 64, 1239 (1990).

[10] K. M. Leung and Y. F. Liu, Phys. Rev. B 41, 10188 (1990).

[11] S. John and R. Rangarajan, Phys. Rev. B 38, 10101 (1988).

[12] E. N. Economu and A. Zdetsis, Phys. Rev. B 40, 1334 (1989).

[13] E. Yablonovitch (unpublished).

[14] A. Scherer, B. P. van der Gaag, E. D. Beebe, and P. S. D. Lin, J. Vac. Sci. Technol. B 8, 28 (1990). 


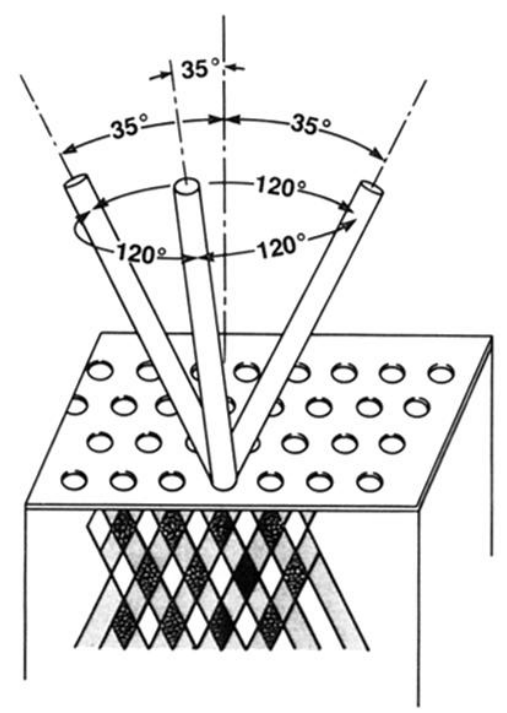

FIG. 2. The method of constructing an fcc lattice of the Wigner-Seitz cells as shown in Fig. 1(b). A slab of material is covered by a mask consisting of a triangular array of holes. Each hole is drilled through 3 times, at an angle $35.26^{\circ}$ away from normal, and spread out $120^{\circ}$ on the azimuth. The resulting crisscross of holes below the surface of the slab, suggested by the cross hatching shown here, produces a fully threedimensionally periodic fcc structure, with unit cells as given by Fig. 1(b). The drilling can be done by a real drill bit for microwave work, or by reactive ion etching to create an fcc structure at optical wavelengths. 